\title{
DEVELOPMENT OF THE MOVABLE MASK FREE FROM TRAPPED MODE FOR THE KEKB
}

\author{
Y.Suetsugu, N.Akasaka, T.Kageyama, Y.Takeuchi, K.Kanazawa and K.Satoh, \\ KEK, Tsukuba, Japan
}

\begin{abstract}
The movable mask is a device that cuts off spent electrons/positrons near the beam orbit and reduces background of the detector [1]. For the KEKB, sixteen movable masks had been installed for each ring. The originally designed masks, however, had severe troubles of heating, arcing and vacuum leaks. Several kinds of improved masks had been designed partially employing RF technologies in dealing with HOM and were installed to the ring step by step. The latest masks are working almost as expected now. Here the problems of movable masks so far are summarized and the structure of the latest movable masks and their status are presented.
\end{abstract}

\section{INTRODUCTION}

Initially sixteen movable masks (or collimators) had been installed for each ring of KEK B-factory (KEKB), i.e., Low Energy Ring (LER) and High Energy Ring (HER) [1]. The movable mask is a device that cuts off spent electrons/positrons just near the beam orbit and reduces background of BELLE detector. The masks were located at the straight and arc sections upstream side of the interaction region (IR). As the stored currents increase, however, the originally designed masks began showing the problems of heating and arcing due to the transient wall current or the intense RF fields of a trapped mode. The heating and arcing sometimes caused vacuum leaks. The trapped mode also excited a strong longitudinal coupled bunch instability and limited the stored beam current. Since the problems were revealed, several improved masks were manufactured and installed step by step. The history of installed masks is presented in Figure 1. The finally installed new masks, Ver.4 and Ver.5, has been working almost as expected up to now. Here we summarize the problems of the old masks (Ver.1 -3), and then report the present status of new ones (Ver.4 and 5).

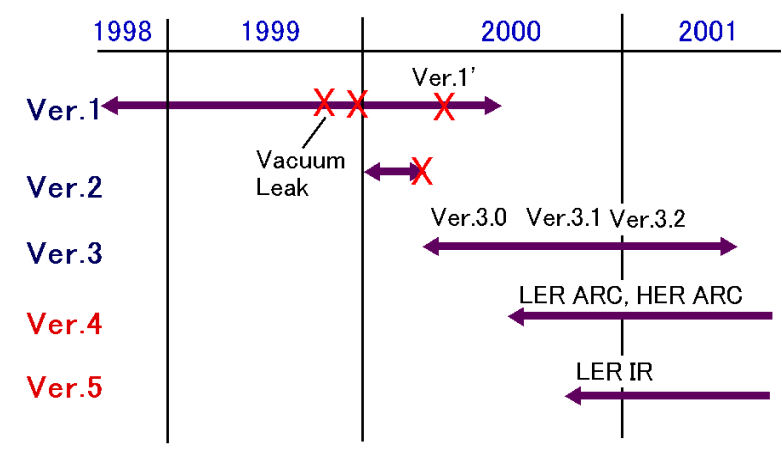

Figure1: History of movable masks

\section{PROBLEMS OF OLD MASKS}

The outlook of the originally designed movable mask (Ver.1) is shown in Figure.2. The mask head is a block made of $\mathrm{Cu}-\mathrm{W}$ and inserted from a side port into the beam chamber. The position of mask head can be controlled remotely observing the background of BELLE and the beam lifetime. The total stroke is about $20 \mathrm{~mm}$ and a vacuum bellows absorbs the movement. The inside of mask head is cooled by water flowing though the stem. The spacer fills the space of port and makes the chamber inner surface smooth. The stem, the spacer and the bellows are made of stainless steel. The fixed and the sliding shield fingers are at the gap between the spacer and the chamber and between the stem and the spacer, respectively. The shield finger is made of silver-coated $\mathrm{Be}-\mathrm{Cu}$. The shield finger should prevent the RF fields from intruding inside the structure. The basic structures for other old masks (Ver.2 and 3) are the same as Ver.1 mask but the mask head is made of pure copper.

The first essential defect of Ver.1 mask is the sliding shield finger. It was so weak to ensure the intense RF fields that it was easily heated and melted. The insufficient cooling structure and the materials with low thermal conductivities worsens the heating problem. Once the sliding shield finger is broken, the RF field can easily enter inside the spacer because of its coaxial line structure. An extensive power of the entered RF field was dissipated at the bellows in a resonance condition. Furthermore, the broken fingers can be a seed of arcing between the spacer and the stem. We had experienced vacuum leaks four times with Ver.1 and Ver.2 masks (see

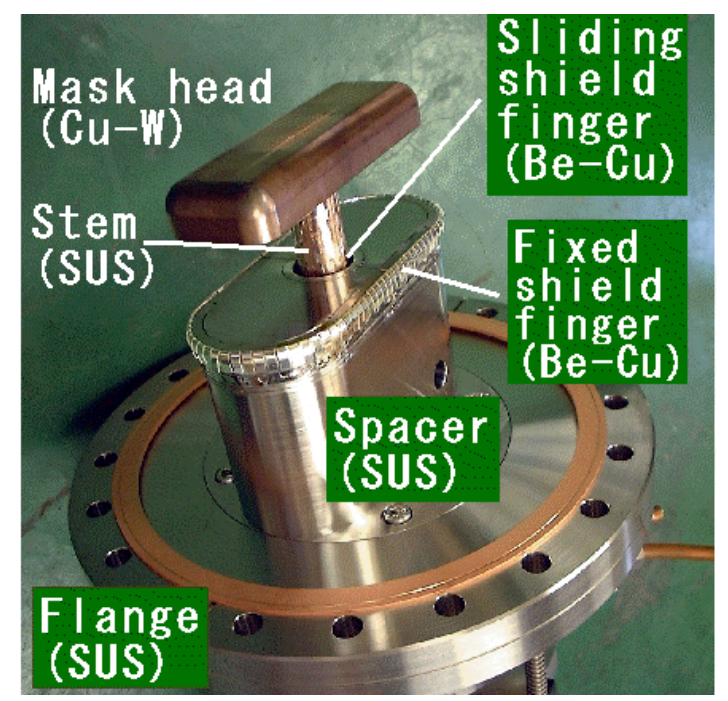

Figure 2: Structure of Ver.1 mask. 


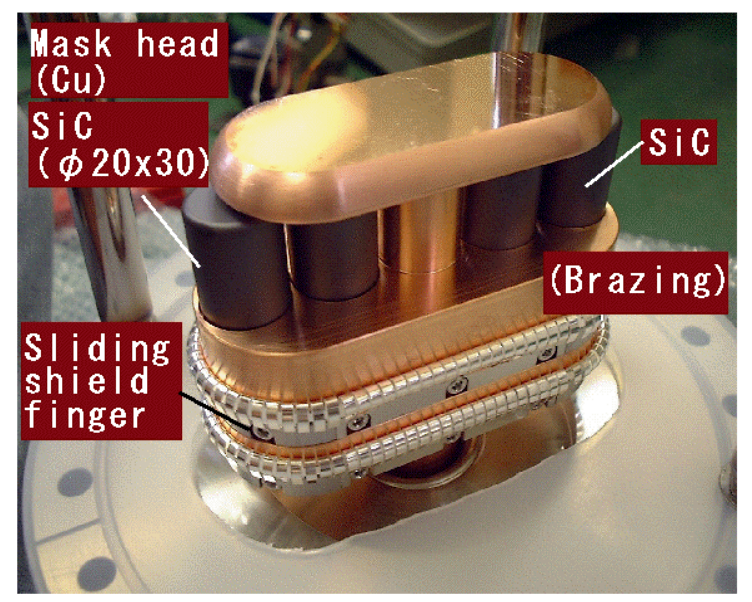

Figure 3: Ver.3.1 mask with SiC HOM absorber.

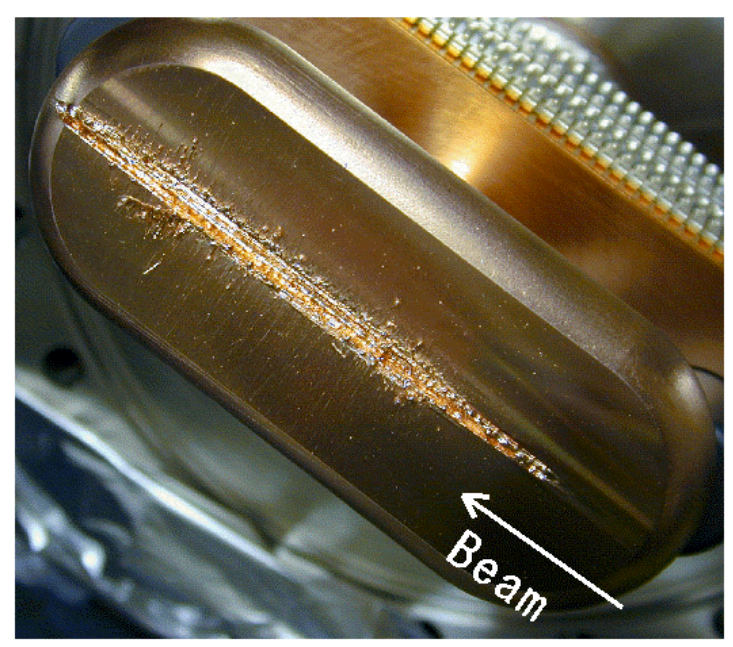

Figure 4: Groove on the mask head.

Figure 1). Two cases are at the gap between the stem and the spacer, and other two cases are at the bellows. For Ver.3 series $(3.0-3.2)$ the sliding shield fingers are moved toward outside as shown in Figure 3 and the current density of it was reduced. The problems of fingers, that is, the excess heating and the vacuum leak, were solved after Ver.3 mask.

The second serious defect of Ver.1, 2 and 3.0 is that there is no effective absorber for the trapped mode of the mask. The Q-values of the major trapped mode of Ver.1 and 2 are several thousands. Even if the sliding fingers are not broken, the intense trapped RF field remains around the mask. Actually, we had sometimes observed a longitudinal coupled bunch instability when the mask head approached the beam. For Ver.3.1 and 3.2, therefore, the $\mathrm{SiC}$ blocks were equipped as a HOM absorber as shown in Figure 3. The Q-value reduced to less than 100. The problem of exciting beam oscillation was settled after Ver.3.1. However, the operation of about 2 months at the beam current over $700 \mathrm{~mA}$ resulted the break of a $\mathrm{SiC}$ block. The absorbed power was estimated as about 500 $\mathrm{W}$ for a SiC block. In this case, the simulation suggests the maximum temperature is larger than 300 degrees at the tip of the block. There is a possibility that the high thermal stress broke the block. The stored beam current of HER, therefore, had been limited by the absorbed power at Ver.3.2 masks.

The third problem, which is still a main problem of new masks (Ver.4 and 5), is the groove generated on the mask head as shown in Figure 4. The groove seems to be made by the main body of the beam when the orbit was accidentally steered or an abnormal beam orbit was set. It is also said that the energy loss due to a power down of an accelerating cavity leads to the vertical beam oscillation or beam size blow up and then causes the digging of mask head. The $R \& D$ to prepare the fast and safe beam abort system is undergoing now. The change of material of the mask head has been also discussed.

\section{NEW MASKS}

\subsection{Ver.4 Mask}

Ver.4 movable mask was at first designed for LER arc section. The structure is quite different from the old ones. The schematic structure of a set of vertical masks and the installed masks in the tunnel are presented in Figure 5 and Figure 6, respectively. The mask head is not a small block as before but a bent chamber (Mask chamber). The driving support moves the chamber and the chamber in itself works as a mask. The chamber is made of pure copper. The total stroke is about $20 \mathrm{~mm}$. The movement of the mask chamber is absorbed by two universal bellows (Bellows chamber) connected at the both side of it. The

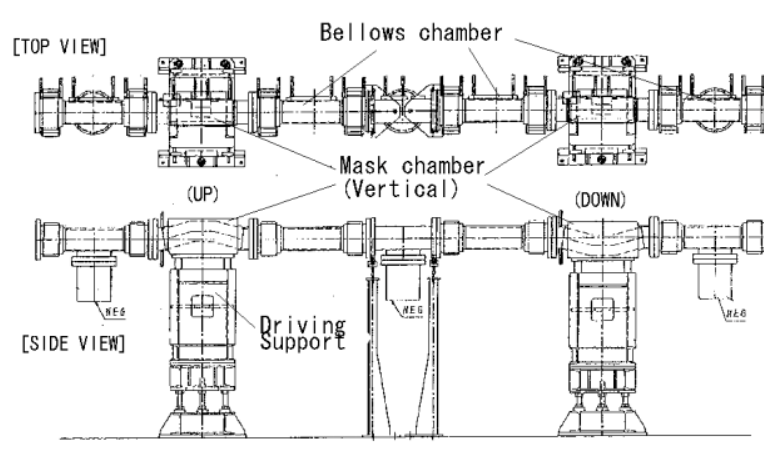

Figure 5: Layout of Ver.4 mask.

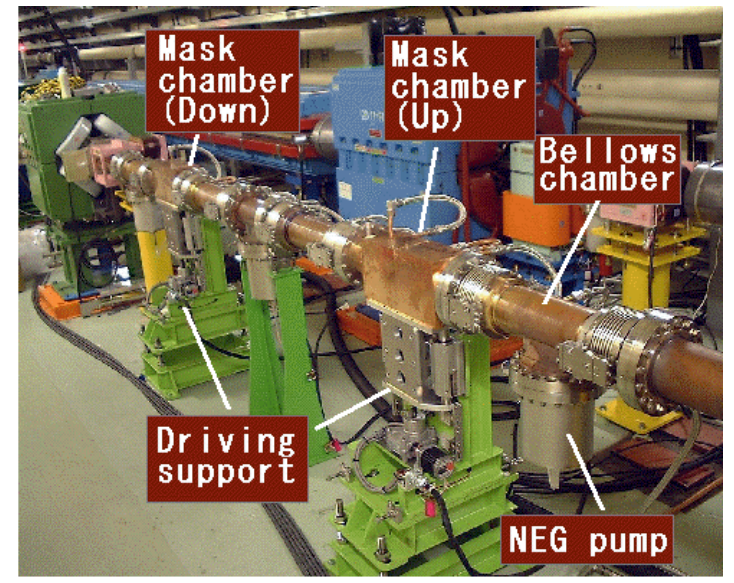

Figure 6: Ver.4 mask installed in the tunnel. 
universal bellows has the RF shield structure same as other bellows for the KEKB [2]. The most important feature of Ver.4 mask is that there is no trapped mode around the mask since the cross section of the beam chamber does not change in this mask region although the HOM is generated. The Ver.4 mask has shown no serious problem in the operation at the beam current over $800 \mathrm{~mA}$ for about one year.

However the temperatures of a corrugation of the bellows located near the masks are higher than others. The temperature depends on the bunch fill pattern, the bunch current and the mask position. The heating shows a resonance phenomenon. The temperature rise, therefore, seems to be due to the HOM (TE mode) generated at the mask chamber. The HOM seems to enter the space between the corrugation and the RF shield finger of a bellows and dissipate the power. The simulation to investigate the heating mechanism and to estimate the power is undergoing. We have to keep watching the temperature as well as the grooves at the mask head.

After the Ver.4 for LER vertical mask, the horizontal mask (4 sets) and HER vertical mask (8 sets) were installed. By installing the HER vertical mask, the limit of the stored current is removed. Further more, Ver.4 for HER horizontal mask will be installed in this year.

\subsection{Ver.5 Mask}

Two Ver.5 masks are developed and installed at the interaction region of the LER. Due to the spatial limitation of the interaction region we cannot use Ver.4 mask there. The structure (cross section) of Ver.5 mask is shown in Figure 7. The basic structure is a plunger used for the tuning of accelerating cavities. The mask head was made of pure copper. The mask damps the RF field heavily through its coaxial wave-guide structure loaded with a cylindrical $\mathrm{SiC}$ absorber (Figure 8). The Q-factor of Ver.5 mask for the major trapped mode is calculated as about 10 and the design power capability of the $\mathrm{SiC}$ absorber is up to $10 \mathrm{~kW}$. Before installation, the mask received a high power test using the $1296 \mathrm{MHz}$ microwave up to $4.2 \mathrm{~kW}$. At the beam current of 500 $\mathrm{mA}$ the absorbed power is estimated as about $800 \mathrm{~W}$. The Ver.5 mask should have no problem, therefore, even for the design current operation at $2.6 \mathrm{~A}$. The grooves on the mask head, however, should be checked regularly. A Ver.5 mask with tungsten head is now manufacturing.

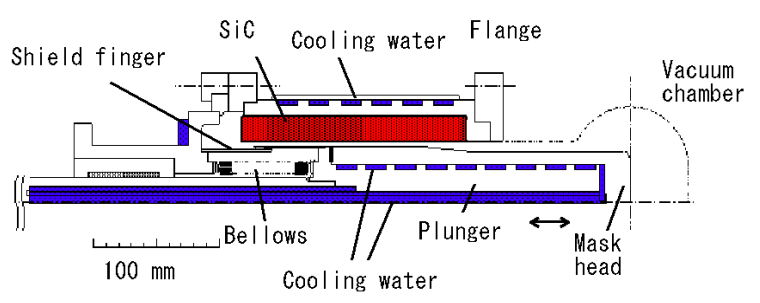

Figure 7: Structure of Ver.5 mask.

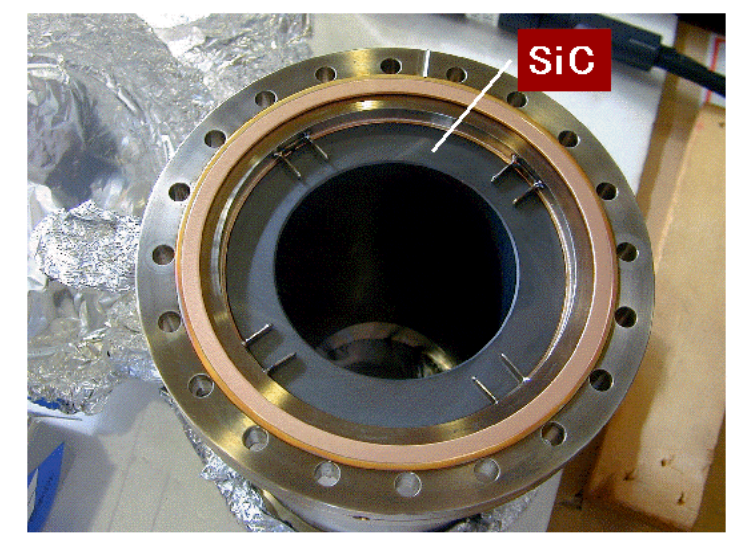

Figure 8: SiC absorber for Ver.5 mask.

\section{SUMMARY}

The old movable masks (Ver.1 - 3) for KEKB has some serious problems in high current operation. The heating trouble and the excitation of the longitudinal coupled bunch instability had limited the stored beam current, that is, the luminosity. The two types of new movable masks (Ver.4 and 5) have been developed to overcome the situation. They were installed to LER and show no essential problem except for some heating at the bellows near the Ver.4 mask. All of movable masks for both rings will be replaced by new ones in this year.

\section{REFERENCES}

[1] Y.Suetsugu et al., "Development of the Movable Mask System for KEKB", in proceedings of EPAC2000, Vienna, June 2000, p.2301.

[2] Y.Suetsugu et al., "Design studies on a vacuum bellows assembly with radio frequency shield for the KEK B factory”, Rev. Sci. Instrum., 67, 2796, 1996. 